\title{
The charged bubble oscillator: Dynamics and thresholds
}

\author{
B. ASHOK ${ }^{1, *}$, THOTREITHEM HONGRAY ${ }^{1}$ and JANAKI BALAKRISHNAN ${ }^{2}$ \\ ${ }^{1}$ Centre for Complex Systems \& Soft Matter Physics, International Institute of Information \\ Technology-Bangalore (IIIT-B), 26/C Electronics City, Hosur Road, Bengaluru 560 100, India \\ ${ }^{2}$ School of Natural Sciences \& Engineering, National Institute of Advanced Studies (N.I.A.S.), \\ Indian Institute of Science Campus, Bengaluru 560 012, India \\ *Corresponding author. E-mail: bashok1@gmail.com, bashok@iiitb.ac.in
}

\begin{abstract}
The nonlinear, forced oscillations of a bubble in a fluid due to an external pressure field are studied theoretically. In the presence of a constant charge on the bubble, the bubble oscillator's behaviour changes markedly. We report results at significantly higher pressures and forcing frequencies than presented earlier. The influence of the bubble's ambient radius on thresholds and dynamics is also reported. Charge and pressure thresholds are calculated for the system, delineating different dynamics.
\end{abstract}

Keywords. Nonlinear oscillations; charged bubble; acoustic forcing.

PACS Nos 05.45.-a; 05.90.+m

\section{Introduction}

Studies on the behaviour of an oscillating gas bubble in an acoustic pressure field in a liquid have long been the focus of great interest to physicists and engineers. Apart from investigations in the context of more spectacular phenomena such as sonoluminescence, studies of bubble dynamics and cavitation have also been of interest in fields like sonochemistry, medical diagnostics, ultrasonic cleaning, design of industrial machinery like pumps and propellors. The published literature on this is detailed and voluminous, and include, to cite a few, Refs [1-7].

On the other hand, it is not often realized that in realistic situations, gas bubbles in liquids such as water carry a net negative charge on them due to the migration of ions in the liquid to the bubble surface. The work of Alty and others [8-11] were some of the first to discuss the presence of charge on gas bubbles in fluids. Forced oscillations of a charged bubble under ultrasonic forcing, on the other hand, were not discussed at any great detail in the literature until our recent work [12-14].

In our earlier papers $[12,13]$ we had modified the Rayleigh-Plesset equation and demonstrated how the presence of electric charge could greatly influence the nonlinear oscillatory dynamics of a bubble in the presence of ultrasound forcing. In our model we assume the charge on the bubble to be uniformly distributed on its surface. In those papers we had confined our studies to applied pressures within the range of approximately 0.4-1.5 atmospheres. In this work, the pressure ranges considered are much wider, around 1-7 atmospheres.

For ultrasound being used in medicine for diagnostic and other purposes, frequencies range from 1 to 15 $\mathrm{MHz}$, with acoustic pressures ranging from 0.5 to 5 $\mathrm{MPa}$ in water. In undersea environments as well, pressure increases by approximately an atmosphere with every 10 metres increase in depth. Our results would hence be of practical interest spanning both the regime of day-to-day medical devices and techniques using ultrasound, as well as more complex systems such as machinery operating at considerable pressures in the sea.

\section{Basic model}

The starting point of our investigations is the modified Rayleigh-Plesset equation which describes the motion and dynamics of a gas bubble in a liquid under an external pressure field. The version employed by Parlitz et al. [15] (and which we subsequently modify) for a gas bubble of radius $R$ and equilibrium radius $R_{0}$ 
is

$\left(1-\frac{\dot{R}}{c}\right) R \ddot{R}+\frac{3}{2} \dot{R}^{2}\left(1-\frac{\dot{R}}{3 c}\right)=\left(1+\frac{\dot{R}}{c}\right) \frac{P}{\rho}+\frac{R}{\rho c} \frac{d P}{d t}$,

with the total pressure $P$ given by

$$
\begin{aligned}
P= & \left(P_{0}-P_{v}+\frac{2 \sigma}{R_{0}}\right)\left(\frac{R_{0}}{R}\right)^{3 \Gamma}-\frac{2 \sigma}{R}-4 \eta \frac{\dot{R}}{R}-P_{\text {stat }} \\
& +P_{v}-P_{s} \sin (\omega t)
\end{aligned}
$$

where $\omega$ is the driving pressure frequency, $P_{s}$ the amplitude of the driving sound pressure, $P_{0}$ the static pressure, $P_{v}$ the vapor pressure, $\sigma$ the surface tension, $\eta$ the dynamical viscosity, $\rho$ the density of the fluid, $c$ the speed of sound in the fluid and $\Gamma$ the polytropic exponent of the gas in the bubble.

When charge is present on the bubble surface, an additional electrostatic pressure term is introduced, while reducing the effective value of the surface tension $\sigma$. The modified equation determining the dynamics of the bubble can then be written as $[12,13]$

$$
\begin{aligned}
& {\left[\left(1-\frac{\dot{R}}{c}\right) R+\frac{4 \eta}{c \rho}\right] \ddot{R}=\frac{1}{\rho}\left(P_{0}-P_{v}+\frac{2 \sigma}{R_{0}}-\frac{Q^{2}}{8 \pi \epsilon R_{0}^{4}}\right)} \\
& \times\left(\frac{R_{0}}{R}\right)^{3 \Gamma}\left(1+\frac{\dot{R}}{c}(1-3 \Gamma)\right)-\frac{\dot{R}^{2}}{2}\left(3-\frac{\dot{R}}{c}\right) \\
& +\frac{Q^{2}}{8 \pi \rho \epsilon R^{4}}\left(1-\frac{3 \dot{R}}{c}\right)-\frac{2 \sigma}{\rho R}-\frac{4 \eta}{\rho}\left(\frac{\dot{R}}{R}\right) \\
& -\frac{1}{\rho}\left(P_{0}-P_{v}+P_{s} \sin (\omega t)\right)\left(1+\frac{\dot{R}}{c}\right)-\frac{R}{\rho c} P_{s} \omega \cos (\omega t) .
\end{aligned}
$$

When a pressure wave is incident on the bubble in the liquid, the difference in pressure causes expansion and rapid collapse of the bubble, followed immediately by smaller afterbounces. This entire sequence of expansion and collapse of the bubble is repeated in each cycle of the ultrasonic forcing. Charge changes the dynamics.

\section{Maximal dimensions of the bubble}

In the original static case, the critical Rayleigh Coulomb-radius for a charged bubble is obtained by balancing surface tension to Coulomb force to get the bubble radius. This does not consider the effects of gas pressure within a bubble nor that of any external forcing. We find that the maximum and minimum bubble radius ( $R_{\max }$ and $R_{\min }$ respectively) are crucially dependent on the charge in the bubble as well as on the driving frequency, and the forcing pressure amplitude $P_{s}$. This is

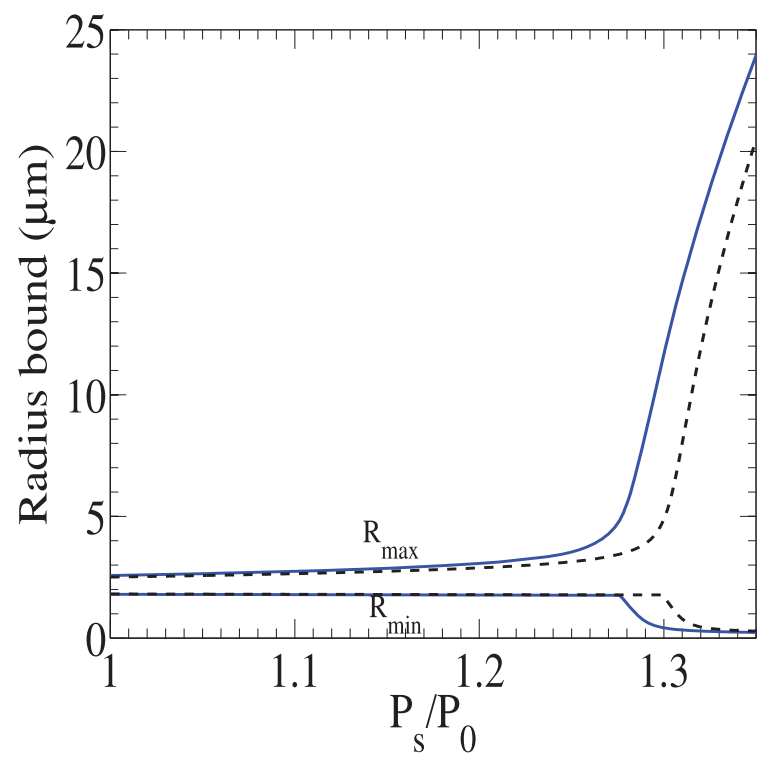

Figure 1. Oscillatory bounds of the bubble: curves showing the minimum radius $R_{\min }$ and maximum radius $R_{\max }$ as a function of driving amplitude $P_{s}$ for a bubble of ambient radius $R_{0}=2 \mu \mathrm{m}$ at driving frequency, $\omega=25 \mathrm{kHz}$. Solid curves shown are a bubble with surface charge $Q=0.14 \mathrm{pC}$, while the dashed lines show the corresponding bounds for the uncharged bubble. The charged bubble has a lower Blake threshold, achieving larger bubble dimensions with a more violent collapse.

shown in figure 1 . In determining the minimum radius of the bubble in the presence of charge or otherwise, one should also keep in mind that contracting to dimensions smaller than the van der Waals radius for the bubble is physically not possible. This, in turn, puts a constraint on the amount of charge the bubble can carry while yet being physically viable.

For larger pressure amplitudes, the bubble oscillations start showing explosive growth. Beyond the Blake threshold, the bubble oscillations are large in amplitude. The dynamics are very sensitive to even small changes in pressures and the Blake threshold essentially demarcates a quasi-static stable oscillating regime from one with highly nonlinear oscillations characterized by explosive growth followed by a sudden collapse $[4,5]$. The distinctly different evolution of the bubble radius for different driving pressure amplitudes can be seen in figure 2. It shows the solution of the bubble radius for three very close amplitudes of the driving pressure $\left(P_{s}=1.42 P_{0}, 1.43 P_{0}, 1.44 P_{0}\right)$ at $20 \mathrm{kHz}$ for $R_{0}=1.5$ $\mu \mathrm{m}$. At $P_{s}=1.42 P_{0}$ the bubble exhibits small amplitude stable oscillations, while even a small increase in pressure amplitude greatly changes the bubble behaviour. In the presence of charge on the bubble, there is a dramatic change in the amplitude of oscillations. This can be seen in figure 3. 


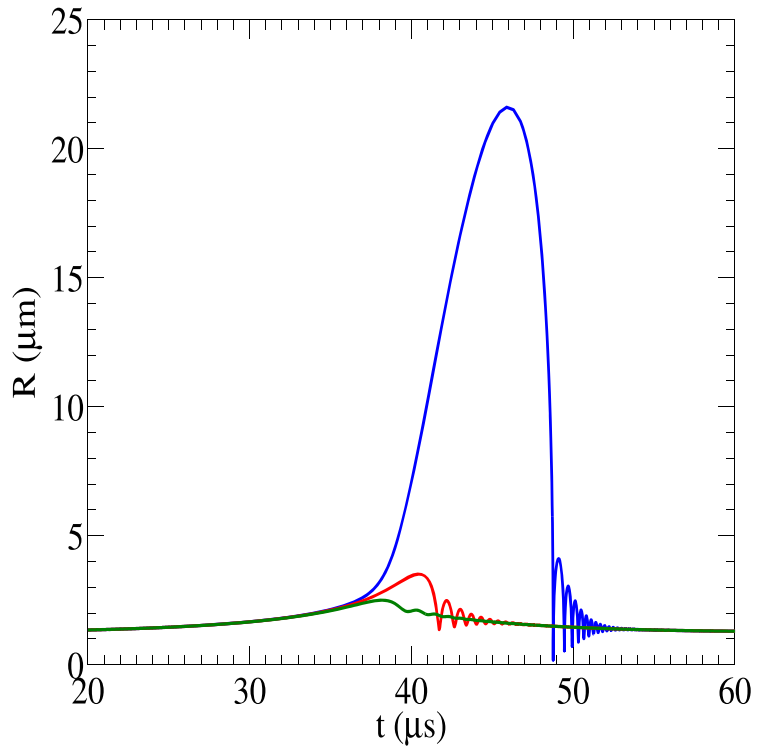

Figure 2. Dependence of bubble radius as a function of time, shown over one cycle, for different driving pressure amplitudes, $P_{s}=1.42 P_{0}$ (green), $1.43 P_{0}$ (red) and $1.44 P_{0}$ (blue). Ambient bubble radius $R_{0}=1.45 \mu \mathrm{m}$, driving frequency $\omega=20 \mathrm{kHz}$. The bubble is uncharged.

To make an estimation of the relative extremal values of the dimensions of the bubble, we define an expansioncompression ratio $\zeta$ that we introduced in earlier work $[12,13]$, defined as

$\zeta \equiv\left(R_{\max }-R_{0}\right) /\left(R_{0}-R_{\min }\right)$

$R_{\max }$ and $R_{\min }$ are the maximum and minimum radii, respectively, of the bubble. A plot of $\zeta$ as a function of the applied pressure amplitude $P_{s}$ is shown in figure 4 . With increasing charge, the curve shifts to the left, while with increasing forcing frequency $\omega$, the curve moves towards the right and downwards. However, at lower pressures, below a critical value of the pressure $\left(P_{f i}\right)$ shown in the figure, the curve becomes independent of the charge and the forcing frequency.

\section{Locating the pressure thresholds}

There are several interesting pressure thresholds that influence the dynamics of a bubble undergoing forced oscillations in a liquid. The Blake threshold is the pressure below which a stable equilibrium bubble can no longer exist, and it is where sudden bubble expansion occurs. This is followed by the upper transient threshold where violent bubble collapse occurs - accompanied by very high bubble radial velocities.

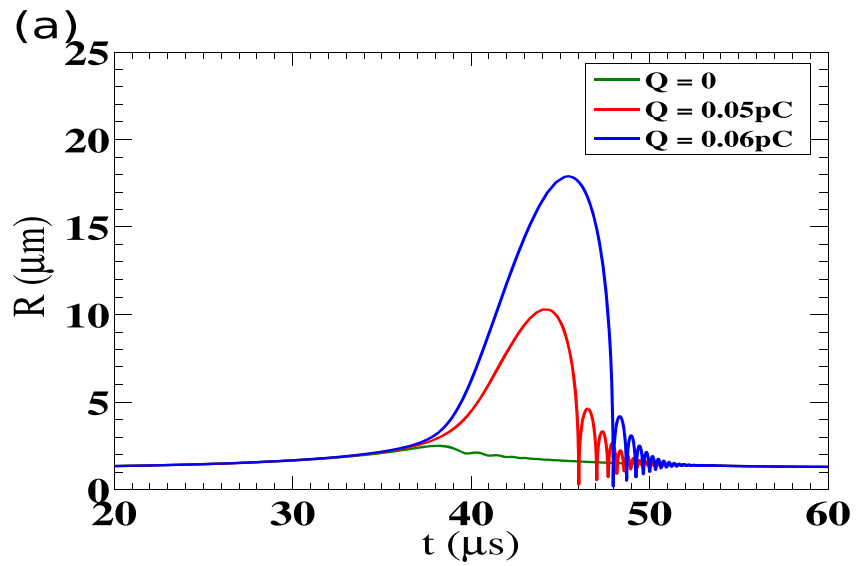

(b)
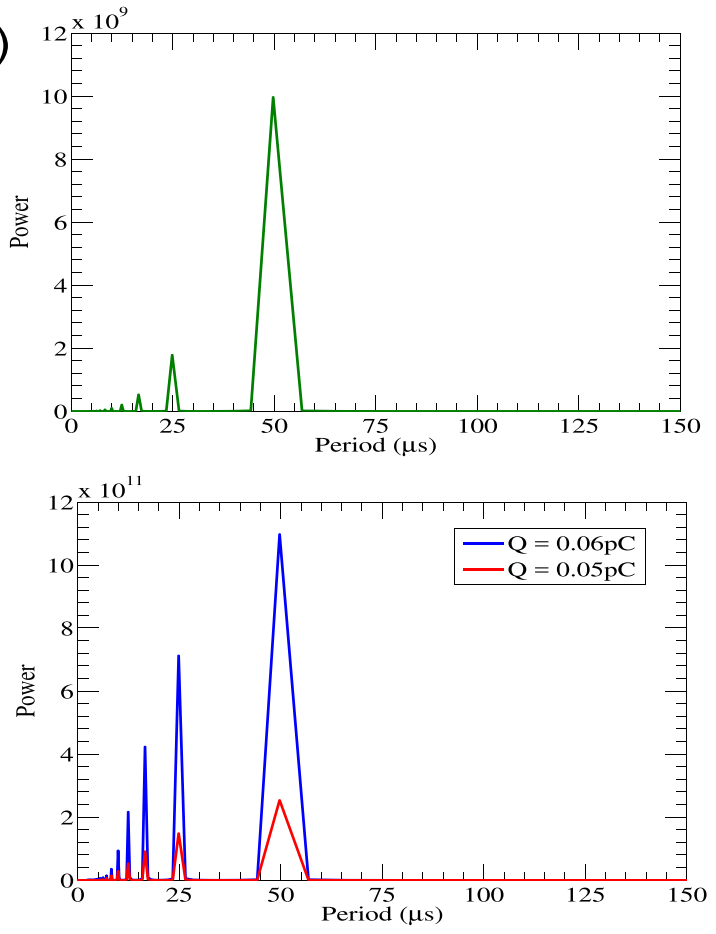

Figure 3. (a) The growth of the bubble at $P_{s}=1.42 P_{0}$ amplitude, driving frequency $v=20 \mathrm{kHz}$, for different values of surface charge $(0$, green; $0.05 \mathrm{pC}$, red; $0.06 \mathrm{pC}$, blue). The behavior of the bubble radius shows dramatic growth even for small inclusion of charge driving the system above Blake threshold. $R_{0}=1.45 \mu \mathrm{m}$. (b) Top: $Q=0$, Bottom: $Q=0.05 \mathrm{pC}$ $\& 0.06 \mathrm{pC}$. Addition of charge for this small driving frequency of $20 \mathrm{kHz}$ does not change the bubble oscillation frequency significantly.

The Blake threshold can be formally defined as

$P_{\text {Blake }} \equiv P_{0}-P_{\mathrm{L}_{\text {crit }}}$.

Here, $P_{\mathrm{L}_{\text {crit }}}$ is a critical liquid pressure corresponding to the critical Blake radius $R_{\mathrm{Blake}} . R_{\mathrm{Blake}}$ is obtained by finding the extremum, with respect to $R$, of the total pressure on the bubble wall due to the surrounding liquid. We have already carried this out in Ref. [12], and 


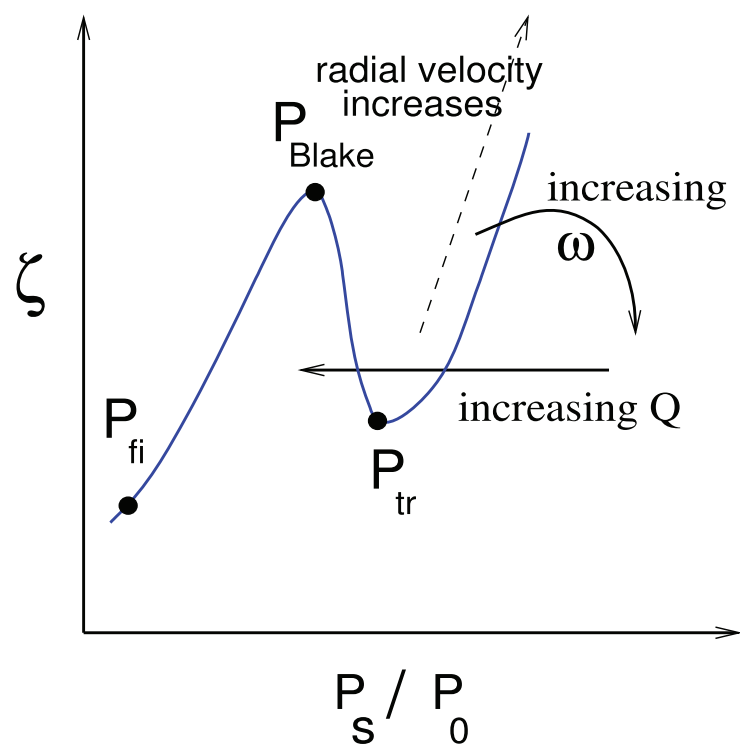

Figure 4. Dependence of the expansion-compression ratio $\zeta$ on the driving pressure amplitude $P_{s}$.

we will merely cite the result here. We find the critical Blake radius and the critical liquid pressure to be

$$
\begin{aligned}
& R_{\text {Blake }}=\frac{1}{2(18 a)^{1 / 6}}\left\{\sqrt{a^{2 / 3}-\frac{10(12)^{1 / 3} \phi}{\sigma}}\right. \\
& \left.\quad+\frac{\frac{6 Q^{2}}{4 \pi \epsilon \sigma} \sqrt{2 a}-\left(a^{2 / 3}-\frac{10(12)^{1 / 3}}{\sigma} \phi\right)^{3 / 2}}{\left(a^{2 / 3}-\frac{10(12)^{1 / 3}}{\sigma} \phi\right)^{1 / 4}}\right\}^{1 / 2}, \\
& a=\frac{9 Q^{4}}{(4 \pi \epsilon \sigma)^{2}}+\sqrt{3}\left(\frac{27 Q^{8}}{(4 \pi \epsilon \sigma)^{4}}+\frac{4000}{\sigma^{3}} \phi^{3}\right)^{1 / 2}, \\
& \phi \equiv R_{0}{ }^{5}\left(P_{0}-P_{v}+\frac{2 \sigma}{R_{0}}-\frac{Q^{2}}{8 \pi \epsilon R_{0}{ }^{4}}\right) \\
& P_{\mathrm{L}_{\text {crit }}}=P_{v}+\frac{\phi}{R_{\text {Blake }}^{5}}-\frac{2 \sigma}{R_{\text {Blake }}}+\frac{Q^{2}}{8 \pi \epsilon R_{\text {Blake }}^{4}} .
\end{aligned}
$$

Here, we have chosen the value of the polytropic index to be $\Gamma=5 / 3$, corresponding to an adiabatic equation of state for the gas.

We find $\zeta$ to be a very useful indicator of the bubble dynamics, far more than the extremal values $R_{\min }$ and $R_{\max }$ alone, or their ratio $R_{\max } / R_{\min }$. This is especially true when we examine $\zeta$ as a function of the applied pressure amplitude $P_{s}$.

As shown in figure 4 , the points of inflection of a plot of $\zeta$ as a function of $P_{s}$ can be identified with the
Blake threshold $P_{\text {Blake }}$ and the upper transient pressure threshold, $P_{\mathrm{tr}}$. As would be expected, when one crosses $P_{\text {tr }}$, there is an increase in the bubble's radial velocity $[12,13]$.

\section{Charge thresholds}

We find that there is a maximal value of the charge $Q_{\max }=Q_{h}$ that a bubble can carry $[12,13] . Q_{h}$ is the value of the electric charge at which the bubble radius reaches the value of the van der Waals hard core radius $h$ for the enclosed gas. This value depends on both the driving pressure amplitude, as well as the driving frequency. The plots shown in figure 5 are for four values of the driving frequency, illustrating the effect that increasing frequency has on the curve. The plateau-like curve for the $Q_{h}-P_{s}$ plots obtained in our earlier work $[12,13]$ is clearly no longer present at higher frequencies. An interesting feature is that at a certain pressure amplitude (approximately 1.61 $P_{s} / P_{0}$ in this figure), all the curves for different frequencies pass through the same point; at this pressure amplitude, $Q_{h}$ is independent of the driving frequency.

Now, as was mentioned earlier, one effect of the presence of charge is to reduce the effective surface tension at the bubble wall. It follows therefore that the equilibrium size of the bubble too would play a role in determining its dynamics.

Indeed, the maximal threshold charge $Q_{h}$ does show a crucial dependence on $R_{0}$. In the present work we

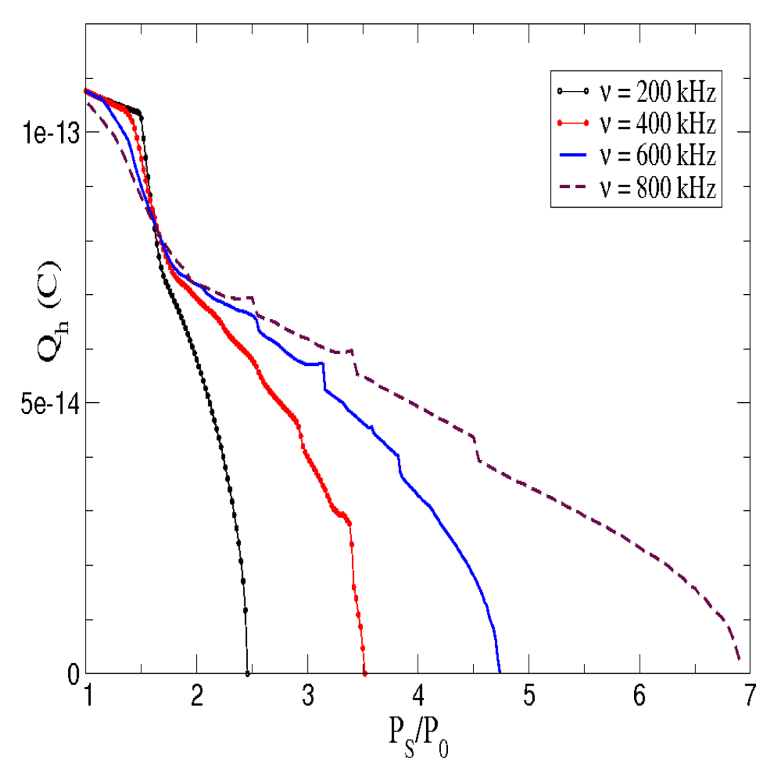

Figure 5. Plot of $Q_{h}$ as a function of $P_{s}$, for a bubble with $R_{0}$ $=1 \mu \mathrm{m}$, at driving frequencies of $200 \mathrm{kHz}, 400 \mathrm{kHz}, 600 \mathrm{kHz}$ and $800 \mathrm{kHz}$. 
have investigated the system at driving frequencies and amplitudes that are an order of magnitude higher than those considered in our earlier work. By studying the system at the first point of inflection of the $Q_{h}-P_{s}$ curve (figure 5) we obtain a power law relating $Q_{h}$ to the ambient bubble radius $R_{0}$. We find that

$Q_{h} \sim R_{0}^{z}$

with $z=1.552$ as seen in figure 6 . Further detailed investigations on the dependence of the dynamics of the bubble on its ambient size are being reported elsewhere [14]. There is also another charge threshold [13] that can be observed, $Q_{\min }$. This is the minimum charge required for a bubble oscillator being driven at a given pressure amplitude $P_{s}$ to attain a maximal radial velocity $\dot{R}=c_{1}$, at a particular driving frequency $\omega$. So, more strictly, this threshold charge should be written as $Q_{\min }\left(c_{1}\right)$, since it is specific for the maximal velocity $c_{1}$. We find that

$Q_{\min }\left(c_{1}\right) \propto\left(\omega-\omega_{h}\right)^{b}$.

Here, $\omega_{h}$ is the frequency at which a bubble at zero charge, at forcing frequency $\omega$, would have a maximum radial velocity of $c_{1}$. While simple arguments along the lines of scaling the kinetic energy with the electrostatic contribution to the energy for a constant maximum radial velocity $c_{1}$ give a value of $b=0.2$, a more systematic approach of linearizing the Rayleigh-Plesset

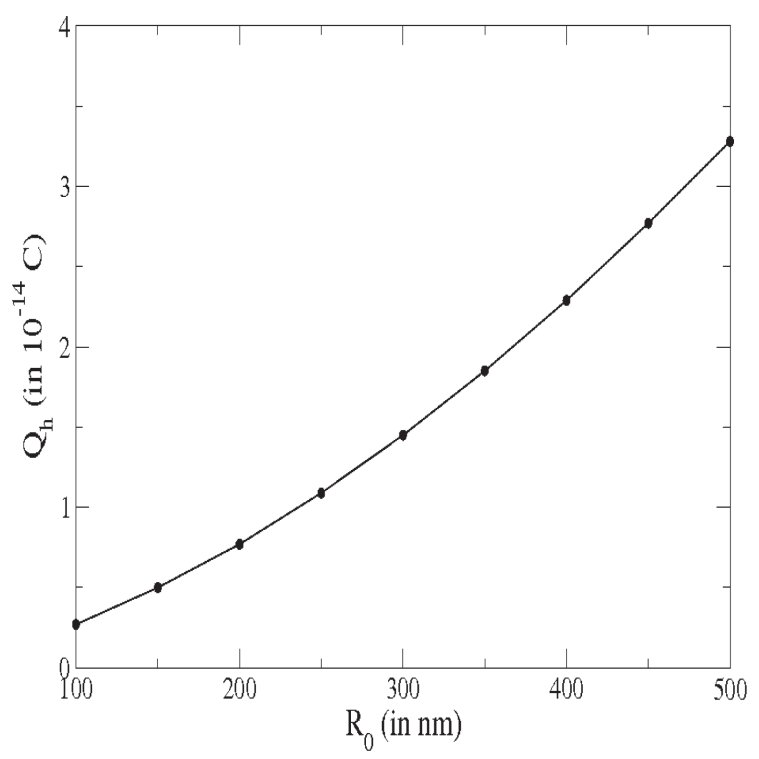

Figure 6. Typical dependence of $Q_{h}$, the maximum permissible charge, on equilibrium bubble radius $R_{0}$, evaluated at the point of inflection of an $R_{h}$ curve at lower forcing frquencies (as shown in figure 5 at $200 \mathrm{kHz}$ ). The inflection point is near (but not necessarily identical to) the Blake pressure threshold. $Q_{h}$ shows a power law dependence on $R_{0}$. equation and considering the driving pressure as a perturbation in the external pressure field yields [13] what is observed from numerical calculations, an exponent which is actually $b=0.25$.

The presence of electric charge can completely change the dynamics of the bubble.

As has been discussed at length in our earlier work [13], the bubble response to the forcing frequency of the applied acoustic pressure wave crucially depends upon the amplitude of the pressure wave as it is a highly nonlinear system, and increasing the driving frequency gives rise to large amplitude oscillations. The presence of charge reduces the effective surface tension, allowing a larger expansion of the bubble in the negative pressure field, leading to a more quick and violent collapse to a smaller minimum radius, as has been explained before in Ref. [13]. It should also be noted that as in our previous work $[12,13]$, our model does not consider shape deformations and assumes only purely radial oscillations. Now these cycles of bubble expansion and collapse accompanied with the afterbounces (the frequencies of recurrence of which is what is being termed as the radial bubble oscillation frequencies) would, at lower driving frequency and amplitudes, typically closely follow the cycle of the external driving pressure wave.

Plots presented in our earlier papers $[12,13]$ showed that the bubble's radial oscillation frequencies did not undergo significant changes on the introduction of charge, at lower driving frequencies, even while there were differences in the amplitudes and phase of oscillations. In fact, in figure 3a, the driving frequency is at the beginning of the ultrasonic range at $20 \mathrm{kHz}$, and still at a comparatively lower value. From figure $3 \mathrm{~b}$ it is seen that the presence of charge does not change the period of oscillations significantly for this driving frequency. However, because of the nonlinearities in the system, this frequency of response of the bubble oscillations changes, yielding other radial bubble oscillation frequencies. This effect is especially magnified at higher pressure amplitudes and driving frequencies, as can be seen by comparing, for example, figure 3 where the driving frequency is only $20 \mathrm{kHz}$ (and $P_{s}=1.42 P_{0}$ ) and figure 7 where the system is being driven at $800 \mathrm{kHz}$ and $P_{s}=2.1 P_{0}$.

Figure 7 shows phase portraits for the system at the higher driving frequency of $800 \mathrm{kHz}$; here, we see that the presence of charge profoundly alters the oscillating frequency of the bubble. As can be seen from figure 7c, more time-periods of oscillation are present for the electrically neutral bubble, than when charge is introduced. Figure 8 is a bifurcation diagram for the maximal bubble radius with charge as the control parameter. The system has a large chaotic regime (for instance around $Q=1.0 \times 10^{-14} \mathrm{C}$; reverse period doubling 

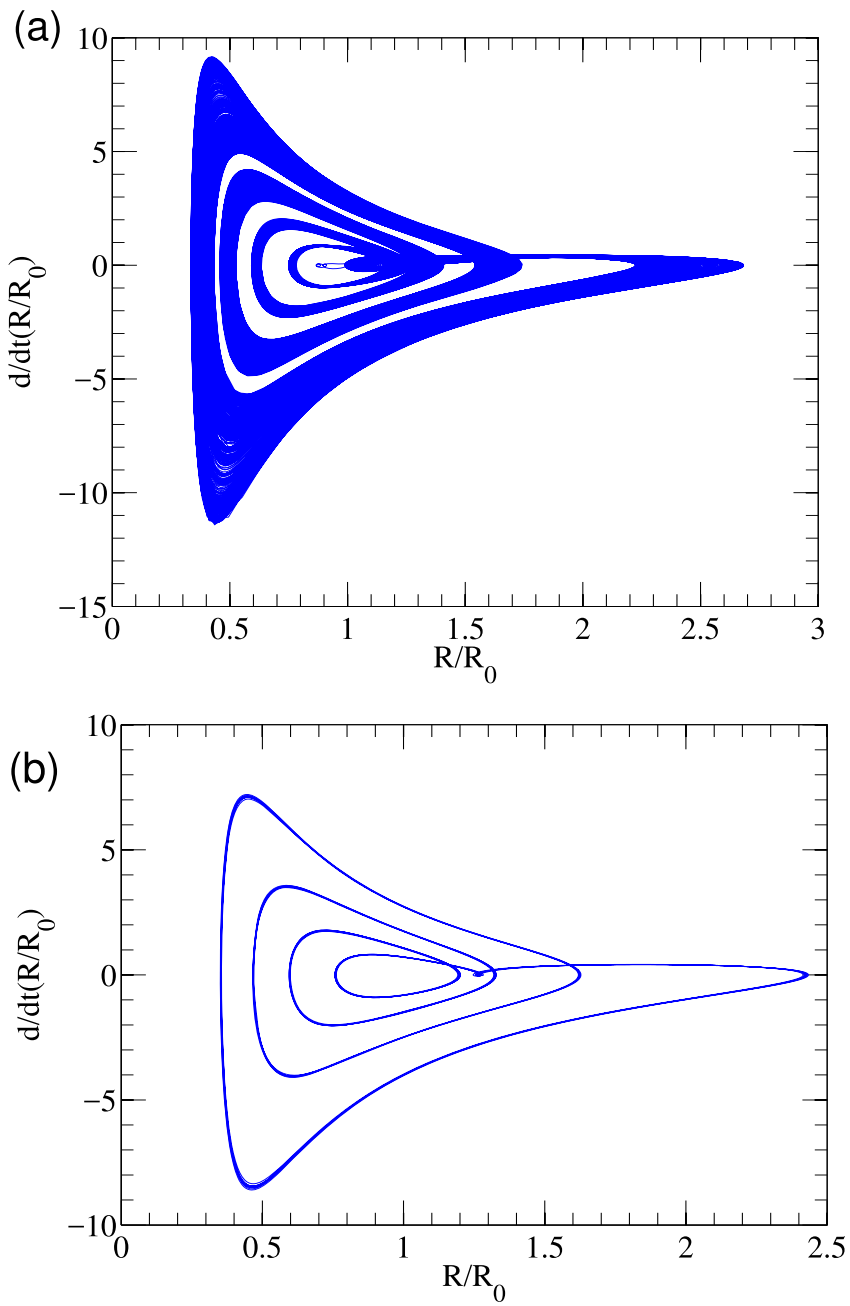

(c)

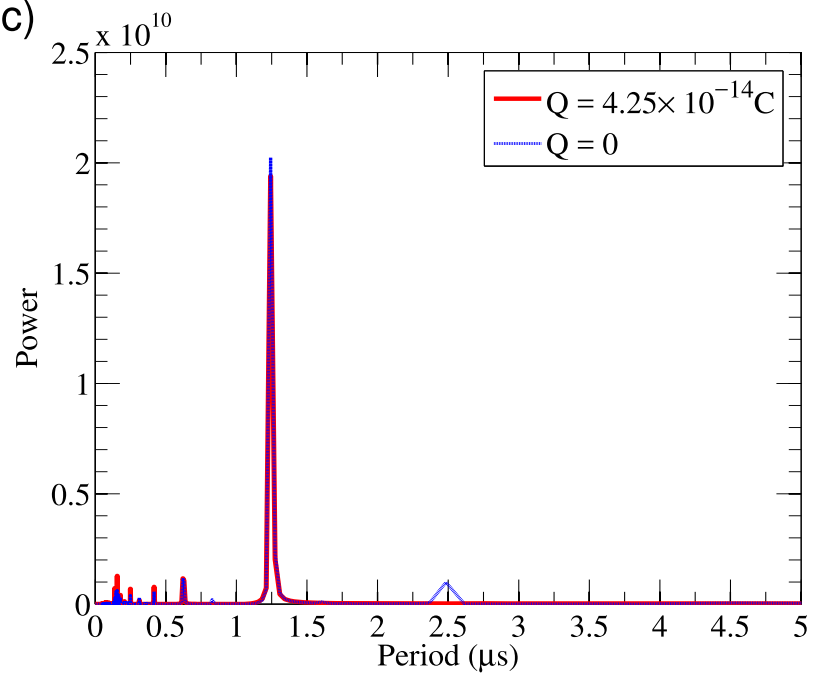

Figure 7. Phase portraits for a bubble of ambient radius $R_{0}=1 \mu \mathrm{m}$, (a) uncharged and (b) with charge $Q=4.25$ $\times 10^{-14} \mathrm{C}$, at $800 \mathrm{kHz}$ driving frequency and $P_{s}=2.1 P_{0}$. (c) Power spectral density of the charged and uncharged bubble showing absence of oscillation modes on introduction of charge, in comparison to the uncharged bubble (note the absence of the sub-harmonic at $2.5 \mu \mathrm{s}$ and the absence of the periods at $0.8 \mu \mathrm{s}$ and $0.35 \mu \mathrm{s}$ ).

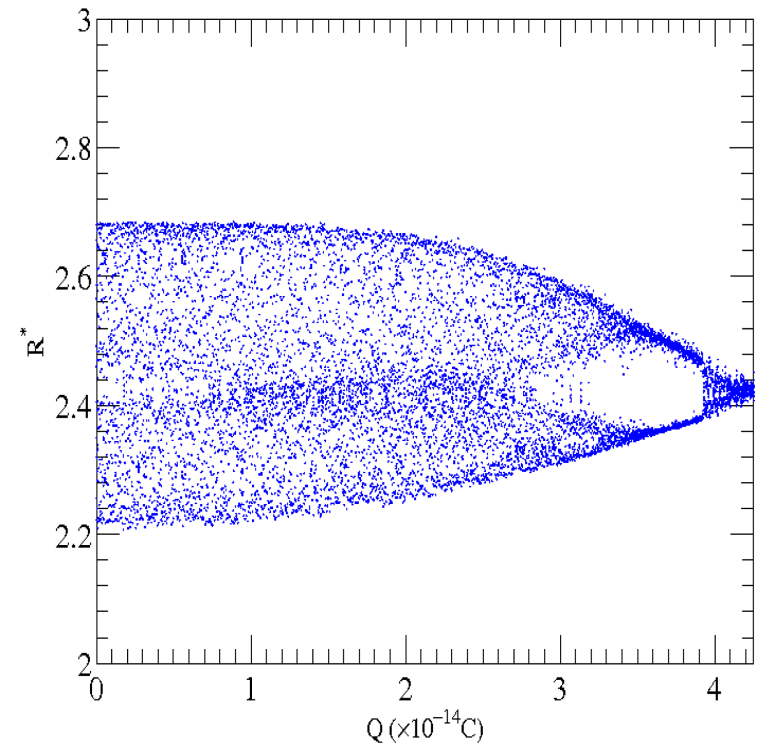

Figure 8. Bifurcation diagram for maximal bubble radius $R^{*}$ with charge $Q$ as control parameter, for $P_{s}=2.1 P_{0}$, at driving frequency of $800 \mathrm{kHz}, R_{0}=1 \mu \mathrm{m}$.

bifurcations are seen at about $Q=3.5 \times 10^{-14} \mathrm{C}$, and at $Q=4.25 \times 10^{-14} \mathrm{C}$, the system shows periodic behaviour.

Lyapunov exponents for the system were calculated in [13] for $R_{0}=1.45 \mu \mathrm{m}$ and $5 \mu \mathrm{m}$, reflecting the rich dynamics exhibited by the bubble oscillator.

We see that for high driving pressures and frequencies considered of the ultrasound, even smaller surface charges (as small in magnitude as $10^{-14} \mathrm{C}$ ) can influence bubble dynamics.

These results are at forcing frequencies which are an order of magnitude higher than those investigated earlier $[12,13]$ and these observations have also not been reported earlier.

\section{Conclusions}

In our work, we have demonstrated how the amplitude of the driving pressure wave as well as its frequency, and also the electric charge present on a bubble undergoing forced oscillations in a liquid, each uniquely determine the nonlinear oscillations and dynamics of the bubble. Comparing with our earlier results $[12,13]$, we find that at higher forcing frequencies, the effect of electric charge on the system's dynamics becomes even more marked, with much smaller magnitudes of surface charge influencing the dynamics of the bubble. The pressure and charge thresholds that exist for the system are also supplemented by effects due to the ambient bubble radius, which tunes their value. The presence of electric charge introduces order in the system which moves 
towards a more periodic regime (which was also seen in figures 7 and 8).

Our methods can also be adapted to study other systems such as formation of bubbles expelled from a nozzle immersed in a fluid under the influence of sound waves $[16,17]$.

\section{References}

[1] L Rayleigh, Philos. Mag. 34, 94 (1917)

[2] M Plesset, J. Appl. Mech. 16, 277 (1949)

[3] M Plesset and A Prosperetti, Ann. Rev. Fluid Mech. 9, 145 (1977)

[4] C E Brennen, Cavitation and Bubble Dynamics (Oxford University Press, New York 1995)

[5] M P Brenner, S Hilgenfeldt and D Lohse, Rev. Mod. Phys. 74, 425 (2002)

[6] J B Keller and M Miksis, J. Acoust. Soc. Am. 68, 628 (1980)
[7] J B Keller and I I Kolodner, J. Appl. Phys. 27, 1152 (1956)

[8] T Alty, Proc. Roy. Soc. (London) 106, 315 (1924)

[9] T Alty, Proc. Roy. Soc. (London) A 112, 235 (1926)

[10] H A McTaggart, Phil. Mag. 27, 297 (1914)

[11] V A Akulichev, Sov. Phys. Acoust. 12, 144 (1966)

[12] T Hongray, B Ashok and J Balakrishnan, Nonlinearity 27, 1157 (2014)

[13] T Hongray, B Ashok and J Balakrishnan, Pramana J. Phys. 84, 517 (2015)

[14] B Ashok, T Hongray and J Balakrishnan, The charged bubble oscillator in the nanometre and micrometre regimes (submitted, 2017)

[15] U Parlitz, V Englisch, C Scheffczyk and W Lauterborn, J. Acoust. Soc. Am. 88, 1061 (1990)

[16] F A C Pereira, E Colli and J C Sartorelli, Chaos 22, 013135-1 (2012)

[17] F A C Pereira, M da Silva Baptista and J C Sartorelli, Phys. Rev. E 90, 042902-1 (2014) 